\title{
Alzheimer's disease and insulin resistance: translating basic science into clinical applications
}

\author{
Fernanda G. De Felice
}

Institute of Medical Biochemistry, Federal University of Rio de Janeiro, Rio de Janeiro, Brazil.

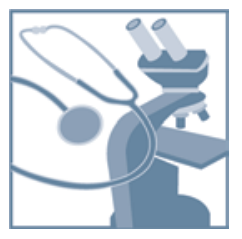

Alzheimer's disease (AD) and diabetes are currently considered among the top threats to human health worldwide. Intriguingly, a connection between these diseases has been established during the past decade, since insulin resistance, a hallmark of type 2 diabetes, also develops in Alzheimer brains. In this article, the molecular and cellular mechanisms underlying defective brain insulin signaling in AD are discussed, with emphasis on evidence that Alzheimer's and diabetes share common inflammatory signaling pathways. I put forward here a hypothesis on how a cross-talk between peripheral tissues and the brain might influence the development of $A D$, and highlight important unanswered questions in the field. Furthermore, I discuss a rational basis for the use of antidiabetic agents as novel and potentially effective therapeutics in AD.

\section{Brain insulin signaling and clinical evidence linking $A D$ and diabetes}

Historically, skeletal muscle, adipose tissue, and liver were regarded as key insulin-sensitive organs involved in insulin-mediated regulation of peripheral carbohydrate, lipid, and protein metabolism. The consequences of impaired insulin action in those organs were deemed to explain the functional and structural abnormalities associated with insulin resistance. On the other hand, since the discovery of insulin in 1922 (1), the brain had been generally considered an insulin-insensitive organ. However, evidence of insulin actions in the brain emerged more than 30 years ago with the demonstration that i.c.v. infusion of insulin decreased food intake in baboons (2). This landmark finding was followed by several studies reporting that the hypothalamic actions of insulin regulate peripheral energy homeostasis $(3,4)$.

The effects of insulin in the brain are not restricted to the hypothalamus, as IRs are widely distributed throughout the encephalon (5). The hippocampus, a region that is fundamentally involved in the acquisition, consolidation, and recollection of new memories, presents particularly high levels of IRs (6), indicating that insulin might have additional targets in the CNS outside of the hypothalamus. Indeed, insulin has been shown to be neuroprotective (7-10) and to affect synaptic plasticity mechanisms $(11,12)$. Insulin has been proposed to regulate neuronal survival and to act as a growth factor (13), possibly by activating IGF receptors (14). In cultured hippocampal neurons, IRs show a punctate dendritic distribution $(15,16)$ consistent with the synaptic occurrence of IRs. IR signaling further regulates circuit function and plasticity by controlling synapse density (17). Nonetheless, knowledge of the precise roles of brain IRs is still limited. While studies indicate memory-improving effects of insulin and learning-associated changes in IR pathways (18-21), specific deletion of brain IRs did not lead to major learning and memory impairment in mice (22). However, compensatory mechanisms may operate to prevent memory deficits in such mice by stimulation of insulin signaling-related pathways via other receptors, including those for IGF and glucagon-like peptide 1

Conflict of interest: The author has declared that no conflict of interest exists. Citation for this article: J Clin Invest. 2013;123(2):531-539. doi:10.1172/JCI64595
(GLP-1). These mechanisms may act to compensate for the absence of IR signaling.

Reflecting a major paradigm shift, recent evidence increasingly indicates that the brain is an important target for insulin actions. The challenge now is to identify in detail the signaling pathways used by insulin in the brain, as failure of those signals has been associated with brain disorders, including stroke, AD, and Parkinson disease (22). The incidence of these neurological disorders appears to be higher in individuals with type 2 diabetes (23), and type 2 diabetes patients can be more than twice as likely to develop AD than nondiabetics (24). In addition to clinical correlations, lower levels of insulin, IGF, and IRs in AD brains further implicate insulin resistance in AD neuropathology (25-27). Brain insulin signaling impairments were also described in animal models of $\operatorname{AD}(10,28)$ and in rodents receiving intracerebral streptozotocin injections (29). A recent elegant study demonstrated that several biomarkers of peripheral insulin resistance are greatly increased in the hippocampus of nondiabetic AD patients (30). As a result of these studies, defective brain insulin signaling is now considered an important feature of AD pathology.

$\mathrm{AD}$ is the most common form of dementia in the elderly, and current estimates suggest it may affect more than 24 million individuals worldwide (31). In addition, recent assessments indicate that about 347 million individuals worldwide have type 2 diabetes (32). Both diseases are common causes of morbidity and mortality, thus underscoring the urgent need to unravel how insulin resistance develops in the brain and peripheral tissues.

\section{Amyloid- $\beta$ oligomers: synaptotoxins that build up in the AD brain and impair neuronal IRs}

In 1906, Alois Alzheimer noticed specific neuropathological alterations in the brain of a female patient who had died as a consequence of an atypical mental illness. Her symptoms included striking memory loss, language problems, and changes in personality. Upon postmortem examination of her brain, Alzheimer found many abnormal proteinaceous deposits, now known as amyloid plaques (33), in the parenchyma. Eight decades later, the chief component of the plaques was determined to be amyloid- $\beta$ (A $\beta)(34,35)$, a peptide that exhibits a high propensity to selfassociate in aqueous medium. 


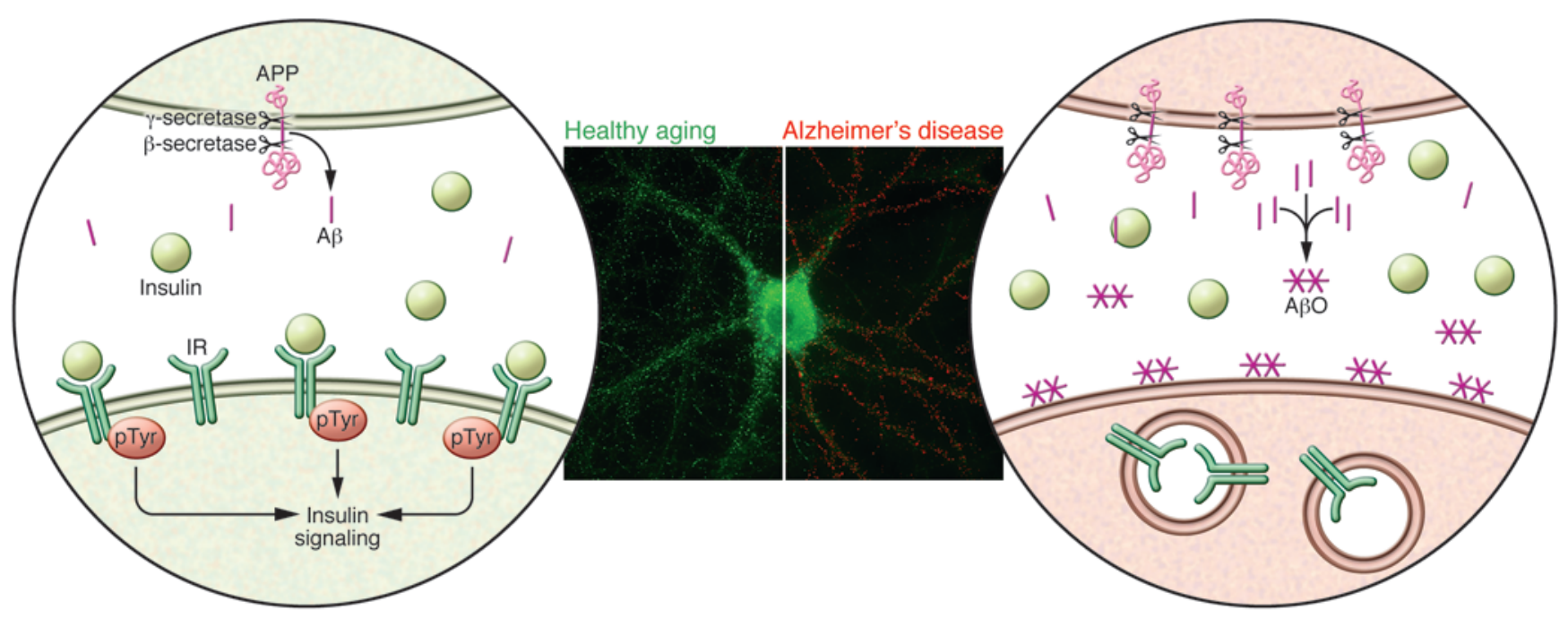

Figure 1

A $\beta$ oligomers remove IRs from the neuronal surface membrane. A composite picture created by merging immunofluorescence images of a control neuron (left image) and a neuron exposed to $A \beta$ oligomers $(A \beta O)$ (right image). Left image: A healthy neuron devoid of $A \beta O$ s (no red puncta observed) presents abundant dendritic IRs (green puncta). A schematic of a dendrite segment is represented in the left circle. Physiological levels of $A \beta$ are produced and there is no accumulation of $A \beta O$ s. The presence of IRs at the surface membrane allows proper insulin signaling and synapse function. Right image: A $\beta O$ binding to neurons (red puncta) causes loss of surface IRs (IR; green puncta), leading to IR internalization $(14,15,47)$. A schematic of a dendrite segment is represented in the right circle: A $\beta O$ s accumulate as a result of elevated $A \beta$ levels generated by cleavage of APP by the $\beta$-secretase (also known as BACE, $\beta$-amyloid precursor cleaving enzyme) and subsequent cleavage by $\gamma$-secretase (a complex consisting of at least 4 components: nicastrin, APH-1, PEN-2, and presenilin). A 30 s attach to a putative receptor complex (not shown; ref. 45) at the neuronal plasma membrane, causing removal of IRs from the membrane and disrupting insulin signaling and synapse function.

Several studies have demonstrated that the neurotoxicity of $A \beta$ requires self-assembly of the $4 \mathrm{kDa} A \beta$ peptide into aggregates of various sizes. The question of which specific aggregate species are responsible for degeneration has been vigorously debated. For decades, large insoluble $A \beta$ fibrils that deposit as amyloid plaques, which can be easily detected in $\mathrm{AD}$ brains, were thought to cause neuronal death (hence, memory loss) in AD. More recent findings, however, indicate that fibrils are probably not the most harmful structures generated by self-association of $A \beta$. Of clinical relevance, individuals who died without any signs of cognitive and intellectual deterioration have been found to present abundant brain amyloid deposits while, conversely, individuals lacking deposits have been found to exhibit varying degrees of cognitive deterioration (36). Moreover, the best correlate of the extent of dementia is not amyloid burden, but rather synapse loss (37), suggesting that synapse deterioration and cognitive impairment could be caused by a toxin other than fibrillar $A \beta$.

William Klein and coworkers first addressed this controversy, showing that $A \beta$ self-aggregates to form neurotoxic-soluble oligomers, aggregates much smaller than fibrils (38) that are not easily observed in neuropathological examination. Oligomers were recently observed at the postsynapse in AD hippocampi (39), and their levels are elevated in the brain and cerebrospinal fluid of $A D$ patients (40-42). Interestingly, the absence of $A \beta$ oligomers at the postsynapse was reported in cognitively intact elderly individuals whose brains presented amyloid deposits (39). Klein's discovery, confirmed and expanded by several groups, led to a novel hypothesis on how $\mathrm{AD}$ progression leads to dementia: synapse failure and neuronal dysfunction are now considered to derive from the impact of $A \beta$ oligomers (43-45).
The impact of $A \beta$ oligomers in the brain appears to be intimately related to defective insulin signaling. The first molecular clue to how the brain might become insulin resistant in $\mathrm{AD}$ came from studies demonstrating that $A \beta$ oligomers bind to hippocampal neurons and trigger the removal of IRs from the plasma membrane (Figure 1) $(15,16)$, which was subsequently verified in $\operatorname{AD}$ brains $(25,46)$. Neurons with oligomers attached to their surface show elevated IR levels in their cell bodies, suggesting a subcellular redistribution of IRs (Figure 1). This leads to decreased responsiveness to insulin, revealed by impaired insulin-induced receptor protein tyrosine kinase activity in cultured neurons exposed to oligomers (15).

Besides IRs, other proteins important for synaptic plasticity, including NMDA- $(47,48)$ and AMPA-type glutamate receptors (49), are removed from the cell surface when neurons are exposed to oligomers, indicating a broad impact on synapses. Oligomer actions are thought to underlie other aspects of brain dysfunction in $\mathrm{AD}$, activating signaling pathways that lead to abnormal tau phosphorylation (50-52) and oxidative stress (53-55), both hallmarks of AD pathology. Thus, altered neuronal IR function is an important aspect of the overall synaptic and neuronal pathology induced by $A \beta$ oligomers. This provides a basis for brain insulin resistance in $\mathrm{AD}$ and is likely connected to impaired learning and memory in disease.

\section{Roles of IRS-1, IRS-2, and IGF-1 signaling in the brain}

IR substrate (IRS) proteins (IRS-1 and IRS-2) play key roles in transmitting signals from the insulin and IGF-1 receptors to several intracellular pathways, including the PI3K/AKT and ERK/MAP kinase pathways, in peripheral tissue $(56,57)$. In contrast, the spe- 
cific roles of IRS-1, IRS-2, and IGF-1 in the brain with respect to cognitive function are still not completely understood, as knowledge of the precise roles of neuronal IRs is at present somewhat limited.

Several reports indicate memory-enhancing effects of insulin, learning-associated changes in IR pathways, and impairment of memory and long-term potentiation (LTP) in diabetic animals (6, $8,11,58)$. IRS- 1 seems to be particularly important for proper brain function, and is found to be inhibited in AD brains $(10,30,46)$ and in an animal model (10). Additionally, attenuation of IRS-1 inhibition is accompanied by improved cognition in transgenic mice (10, $52)$. On the other hand, IRS-2 signaling impairs dendritic spine formation (59). Deletion of IRS-2 further reduces amyloid deposition, cognitive deficits, and premature mortality in a transgenic mouse model of AD (60). The beneficial effects of IRS- 2 deletion in $\mathrm{AD}$ pathology parallel the effects on lifespan, with less IRS- 2 signaling resulting in extended lifespan in mice (61). IRS-2 levels are also decreased in the amyloid precursor protein-presenilin 1 (APP/ PS1) transgenic mouse model of AD (10) and in AD brains (46), which may indicate a compensatory mechanism to decrease IRS-2 signaling (62). However, whether this is an active neuroprotective response or a secondary response to the neurodegenerative process remains unclear. Recently, physiological doses $(1 \mathrm{nM})$ of insulin and IGF-1 were demonstrated to activate different IRS signaling pathways in ex vivo AD brain slices (30); impaired IR signaling was associated with dysfunctional IRS-1, while IGF-1 resistance was associated with dysfunctional IRS-2 signaling (30). Therefore, an apparent dichotomy exists between the neuroprotective effects of brain insulin signaling and its deleterious actions on lifespan and memory. Conceivably, IRS-1 positively regulates memory, while IRS- 2 acts as a negative modulator of memory formation, but the roles of neuronal IRS-1, IRS-2, and IGF signaling in health and disease certainly need further exploration.

\section{Molecular basis for brain insulin resistance in AD}

As several pathological features, including impaired insulin signaling and inflammation, appear to be shared by diabetic and $\mathrm{AD}$ patients, mechanisms analogous to those that account for peripheral insulin resistance in type 2 diabetes likely underlie impaired brain insulin signaling in AD. Indeed, recent findings link pathogenic mechanisms triggered by $A \beta$ oligomers in $A D$ brain to mechanisms present in diabetes $(10,27,52)$. In type 2

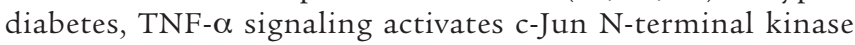
(JNK) (63). This results in IRS-1 serine phosphorylation (IRS$1 \mathrm{pSer})$, blocking downstream insulin signaling and triggering peripheral insulin resistance (15). Similarly, A $\beta$ oligomers cause abnormal activation of the TNF- $\alpha / J N K$ pathway and IRS- 1 inhibition in cultured hippocampal neurons $(10,52)$. JNK activation and IRS- 1 inhibition are further present in the hippocampi of cynomolgus monkeys given i.c.v. injections of $A \beta$ oligomers, as well as in the brains of a transgenic mouse model of AD (10). Likewise, postmortem AD brains showed elevated IRS-1pSer $(10,30)$ and activated JNK levels $(10)$. Because oligomers instigate internalization and redistribution of neuronal IRs (15), IR removal from the cell surface may underlie, or facilitate, IRS1 pSer. Insulin consistently blocks both IR downregulation (16) and IRS- 1 pSer induced by A $\beta$ oligomers (10).

Downstream of IRS-1 and PI3 kinase, oligomers induce inhibitory phosphorylation of AKT, a central signaling molecule of the IR pathway. Elevated AKT-pSer473 levels are associated with inflammation and peripheral insulin resistance $(64,65)$. Notably, stimulation of AKT-pSer473 by oligomers occurs whether or not insulin is present (15), suggesting the involvement of a pathway independent of IRs, and possibly involving TNF- $\alpha$ signaling. Recently, elevated TNF- $\alpha$ and APP levels were observed in the hippocampus of a mouse model of high-fat diet-induced obesity (66), mechanistically linking the pathophysiology of obesity to AD.

In peripheral insulin resistance, TNF- $\alpha / J N K$ activation is linked to major inflammatory and stress signaling networks, including ER stress and the stress kinases IKB kinase (IKK) and protein kinase regulated by RNA (PKR) $(67,68)$. Interestingly, IKK and PKR appear to mediate oligomer-induced neuronal IRS-1 inhibition (10). Therefore, ER stress, which is reported to occur in AD brains (69), likely further underlies $A \beta$ oligomer-induced defective neuronal insulin signaling. If confirmed, this observation would reinforce the notion that common mechanisms underlie impaired peripheral insulin signaling in type 2 diabetes and brain insulin resistance in $\mathrm{AD}(10)$.

\section{Oxidative stress: a link between AD and diabetes}

ROS are minor cytotoxic products of normal mitochondrial metabolism. In the brain, the transient production of ROS plays a role in synaptic signaling, with ROS acting as messenger molecules in LTP (70). Similarly, moderate ROS levels are thought to enhance peripheral insulin sensitivity (71). However, the imbalance between mitochondrial ROS production and intracellular levels of antioxidant defenses leads to mitochondrial dysfunction, abnormally elevated ROS levels, and oxidative stress, which is associated with both peripheral insulin resistance and $\operatorname{AD}(71,72)$. The transcriptional activity of FoxO proteins has been proposed to underlie defective insulin signaling in $\mathrm{AD}$ and diabetes, as insulin resistance and oxidative stress may promote the FoxO response that leads to JNK activation (73).

Interestingly, $\mathrm{A} \beta$ oligomer-induced neuronal oxidative stress $(53,54,74)$ is blocked by insulin $(16,75)$. The mechanism of protection by insulin appears to involve activation of AKT (75) and prevention of abnormal NMDA receptor (NMDA-R) activation (76). NMDA-R dysfunction indeed seems to play a role in oxidative stress and defective neuronal insulin signaling in $\mathrm{AD}$, as $A \beta$ oligomer-induced inhibition of IR signaling is prevented by the NMDA-R blocker memantine (15). Memantine and an anti-NMDA-R antibody further attenuate the oligomer-induced increase in intraneuronal calcium, which is essential in causing neuronal oxidative stress $(53,74)$. A mechanism involving aberrant calcium influx and oxidative stress may underlie insulin resistance in $\mathrm{AD}$, as glutamate and neuronal depolarization reduce the responsiveness of IRs to insulin (15), and chelation of intracellular calcium with BAPTA-AM prevents both oligomer-induced IR inhibition (15) and oxidative stress (53). NMDA-R signaling itself is affected by $\mathrm{A} \beta$ oligomers (48). These effects are concordant with existing IR regulatory mechanisms and suggest a possible physiological feedback between neuronal activity and insulin signaling. Thus, abnormal ROS levels may trigger a vicious cycle that impairs insulin signaling in AD.

\section{A cumulative hypothesis for AD}

Appealing hypotheses have been proposed to explain how AD develops, undoubtedly providing major contributions to our understanding of $\mathrm{AD}$ pathogenesis. The revised amyloid cascade hypothesis (77), which includes $A \beta$ oligomers as synaptotoxins in $\mathrm{AD}$, has received major attention. In familial forms of $\mathrm{AD}$, muta- 


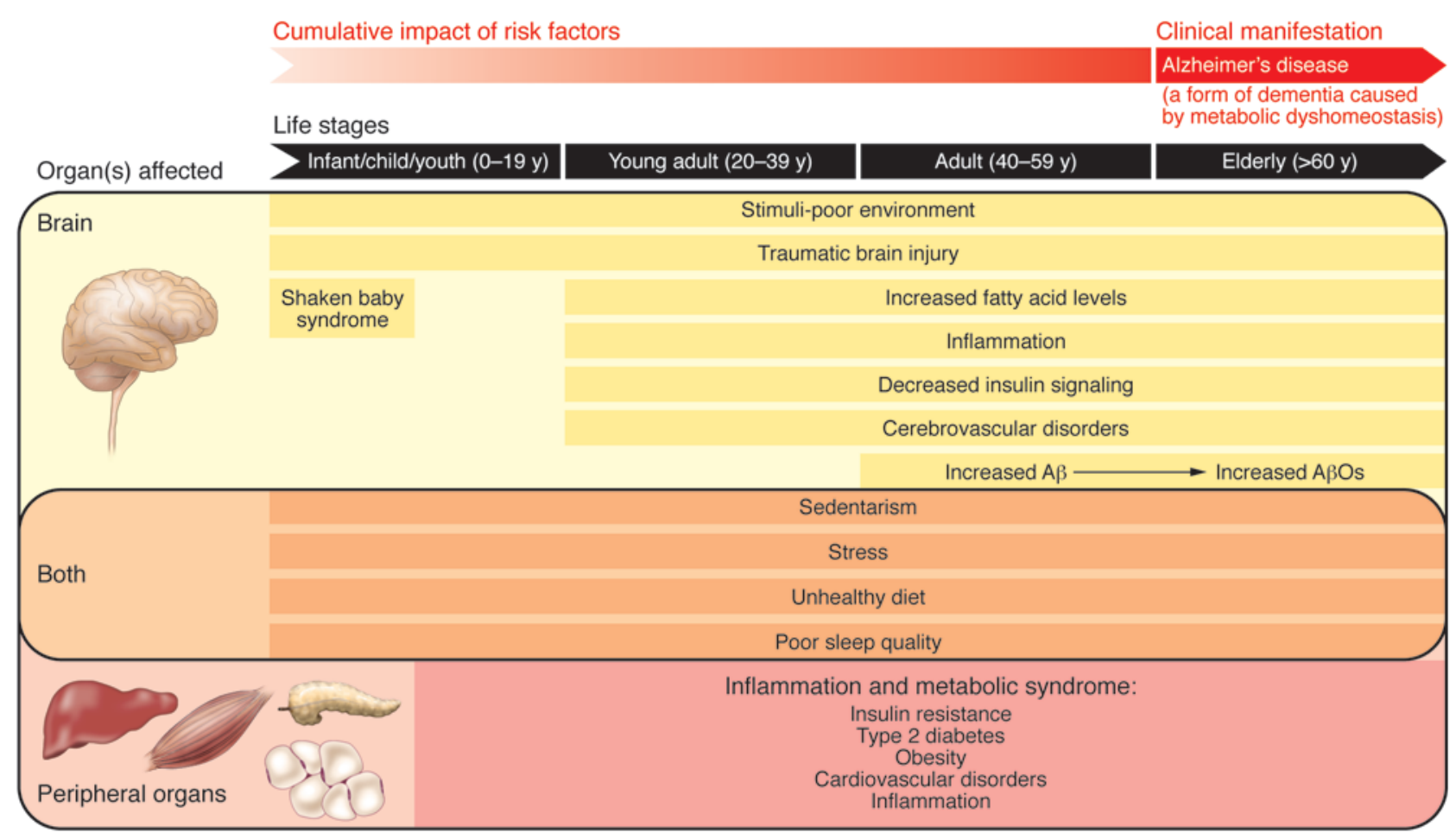

Figure 2

A "cumulative hypothesis" for development of sporadic AD. Listed are the different types of injuries that may impact the brain (yellow), peripheral organs (pink), or both systems (orange) throughout life and increase the risk of sporadic AD. The cross-talk between brain and peripheral tissues may eventually result in defective brain metabolic homeostasis, which might be closely linked to elevated $A \beta$ production and progressive accumulation of $A \beta O$ s in the brain. $A D$, which could thus be considered a form of dementia caused by metabolic dyshomeostasis, would manifest in the elderly as a result of the cumulative, lifelong impact in the peripheral tissues and the brain.

tions in the APP and/or presenilin genes lead to increased $\mathrm{A} \beta$ production (78), strongly suggesting a causative relationship between $\mathrm{A} \beta$ generation and pathogenesis in $\mathrm{AD}$. However, in sporadic $\mathrm{AD}$ (which corresponds to greater than $90 \%$ of AD cases), the exact mechanism that leads to oligomer-amyloid accumulation in AD brain remains a mystery. The discovery that insulin resistance develops in AD brains might be seen as an additional complication, because insulin resistance itself is a complex metabolic disorder (79). Nevertheless, this new link between AD and diabetes may in fact shed light on how sporadic AD develops.

Here, an alternative hypothesis is proposed in which a cross-talk between brain and peripheral tissues plays a central role in triggering the onset of sporadic AD (Figure 2). Similar hypotheses have been put forward based on work from Craft and de la Monte (80, 81), providing important advances in elucidating the connection between $\mathrm{AD}$ and diabetes. In the hypothesis presented here, I highlight the central role of peripheral inflammation in causing sporadic $\mathrm{AD}$ and propose that such events can start very early in life.

An unhealthy lifestyle (e.g., lack of or insufficient physical activity, inadequate nutrition), which may start in the first years of life and increase the prevalence of type 2 diabetes in youth (82), might have an important role in susceptibility to AD later in life, as pointed out by Mark Mattson (83). An unhealthy lifestyle triggers deleterious processes in peripheral tissue, leading to the activation of pathways related to chronic metabolic syndrome (including obesity, insulin resistance, and type 2 diabetes). Notably, an unhealthy lifestyle exerts several detrimental effects on brain aging (84). Furthermore, poor sleep quality, as occurs in aging and in many obese individuals, may contribute to an increased risk of type 2 diabetes and $\operatorname{AD}(85,86)$. According to this proposal, a cumulative impact on peripheral organs eventually results in defective brain metabolic homeostasis (see below), ultimately leading to AD (Figure 2).

In addition, different types of injuries may impact the brain and/ or peripheral organs throughout life. Such events may start very early in life, with stress caused by maternal separation or physical or emotional child abuse, including shaken infant syndrome associated with cerebral contusions. Although the long-term outcomes of mild brain injury in infants still need to be fully understood, learning difficulties and memory problems have been reported to occur in affected children (87). Mild brain injury accelerates $\mathrm{A} \beta$ deposition, tau pathology, and cognitive deficits in transgenic $\mathrm{AD}$ mice $(88,89)$ and further leads to neurodegeneration and cognitive deficits in immature rats (90). In any period of life, traumatic brain injury may further lead to $\mathrm{AD}$, as evidenced by elevated production of $A \beta$ in such events (91). Additionally, brain insulin signaling declines with age (92). This deficiency may be a consequence of decreased insulin uptake into the brain following sustained peripheral hyperinsulinemia (93). This point, however, is still somewhat unclear, as an earlier study reported that AD patients have elevated insulin levels in their cerebrospinal fluid (CSF) under fasting conditions (94). However, more recent studies demonstrated that CSF insulin levels are decreased in patients with mild AD (95). Furthermore, a high saturated fat and high 
glycemic diet was found to lower CSF insulin concentrations in healthy adults (96), corroborating the possibility that physiological mechanisms result in decreased brain insulin levels following peripheral hyperinsulinemia. Interestingly, impaired insulin sensitivity has been linked to cognitive deficits and structural and functional brain deficits in the elderly (97).

Inflammation plays a key role in metabolic disorders, particularly in obesity and type 2 diabetes (98). It is important to consider the causes of inflammation in peripheral tissues and to determine how this process could impact the brain, as activation of inflammatory signaling pathways is closely linked to the development of ER stress and insulin resistance $(79,99)$. A common pathway leading to peripheral insulin resistance involves lipid accumulation (e.g. palmitic acid, ceramides) in liver and skeletal muscle $(100,101)$. Prolonged consumption of food rich in saturated fatty acids is thought to be deleterious, as saturated fatty acids exert adverse health effects and are more likely to cause peripheral insulin resistance than unsaturated fatty acids. In fact, saturated fatty acids activate JNK and induce peripheral insulin resistance (102), abnormalities that are also observed in $\mathrm{AD}$ brains $(10,30)$. Interestingly, enhanced brain fatty acid uptake and accumulation have recently been reported in patients with metabolic syndrome, a process reversed by weight reduction (103). Also, ceramides, which are generated in the liver, have been proposed to cross the blood-brain barrier and cause brain insulin resistance and neurodegeneration (81). A mechanism linking lipid homeostasis and $A \beta$ production was recently demonstrated, as microRNAs were found to regulate an enzyme involved in ceramide synthesis and, in turn, $A \beta$ generation (104). Furthermore, cholesterol depletion inhibits $A \beta$ generation (105), and growing evidence suggests that other lipids may have important roles in AD (106).

Moreover, microglial activation and inflammation-mediated neurotoxicity are suggested to be important in the pathogenesis of $\mathrm{AD}(107,108)$. As disease progresses, $\mathrm{A} \beta$ deposits, neurofibrillary tangles, and damaged neurons, along with brain insulin resistance and ER stress (109), are thought to provide feedback stimuli for inflammation.

Thus, inflammatory processes related to insulin resistance, such as inflammation caused by an unhealthy diet and physical inactivity, likely play a decisive role in linking peripheral and brain dyshomeostasis. The accumulation of and imbalance in certain lipids in peripheral tissues could lead to their continued release into the systemic circulation and their subsequent transport across the blood-brain barrier, which may affect the brain. In fact, nutritional inflammation impacts the hypothalamus $(110,111)$. Therefore, in brain regions that are affected in AD (e.g., hippocampus, frontal cortex), inflammation could lead to elevated $A \beta$ production and to the progressive accumulation of $\mathrm{A} \beta$ oligomers in the brain. $\mathrm{AD}$, which could thus be considered a form of dementia caused by metabolic dyshomeostasis, would manifest in the elderly as a result of this cumulative, lifelong impact on the periphery and the brain (Figure 2).

\section{Stimulation of brain insulin signaling as a therapeutic approach in AD}

$\mathrm{AD}$ is a catastrophic disease that demands effective treatment. Molecular links between dysregulated insulin signaling in AD and diabetes (10) raises the prospect for novel therapeutic strategies for AD based on antidiabetes agents. Conversely, diabetes therapy may affect the brain in other ways, as disruptions in the mechanisms of hypothalamic nutrient sensing alter homeostatic responses and contribute to the pathophysiology of obesity and type 2 diabetes (110).

Brain insulin signaling declines with age (92), a major risk factor for $\mathrm{AD}$, suggesting that the beneficial effects of insulin signaling could be extended to $\mathrm{AD}$ patients. In agreement with the proposed role of insulin signaling in brain regions associated with cognition, especially in the hippocampus (5), intranasal insulin treatment improves memory in healthy adults, without changing blood levels of insulin or glucose $(19,112)$. Intranasal insulin administration further regulates peripheral energy homeostasis in humans $(113,114)$.

Moreover, intranasal insulin enhances verbal memory in memory-impaired subjects (20) and, importantly, improves performance in early $\mathrm{AD}$ patients $(18,19)$. Beneficial effects on cognitive functions have been reported for acute and long-term intranasal insulin administration. Although potential adverse side effects might be expected from the use of intranasal insulin (115), no treatment-related severe adverse events occurred in a 4-month period of treatment, and most adverse events were minor, such as mild rhinitis (21). The beneficial effects of acute insulin administration were not observed in APOE $\varepsilon 4$ carriers $(116,117)$, who have a higher risk for developing $\mathrm{AD}$, but further studies are needed to confirm this association.

The mechanism of protection by insulin seems to involve downregulation of sites where $A \beta$ oligomers bind (16), consequently preventing synapse loss, IR internalization, oxidative stress, and impairment of synaptic plasticity $(16,118)$. Notably, insulin fails to block oligomer binding to neurons when IR tyrosine kinase activity is inhibited (16). The protective role of insulin thus derives from IR signaling-dependent downregulation of oligomer-binding sites in neuronal processes, indicating the occurrence of cellular mechanisms that physiologically protect synapses against $A \beta$ oligomers (16). Such active synaptic protection mechanisms could contribute to preserved cognitive function in normal individuals, while impaired mechanisms might render neurons vulnerable to oligomer-induced synaptotoxicity. Indeed, insulin may have important therapeutic implications in early and/or intermediate phases of $\operatorname{AD}(20,119)$, preventing oligomer binding and blocking the pathology of IRs in cultured neurons $(15,16)$ and in AD brains (46). However, at later disease stages, when surface IRs dwindle, insulin might stimulate other receptors (e.g., IGF-1R) and thus improve AD-related deficits.

Nevertheless, alternative approaches to bypass IRs and boost insulin-related signaling pathways might provide an improved therapeutic approach or an addition to insulin-based therapies in AD. Glucagon-like peptide-1 receptor (GLP-1R) agonists are an attractive option because they activate pathways common to insulin signaling through G-protein-dependent signaling (120). GLP-1Rs are present and functional in cultured neurons as well as in rodent and human brains $(121,122)$. Exendin-4 and liraglutide are GLP-1R agonists that were recently approved for the treatment of type 2 diabetes. In mice, GLP-1R analogs are stable in blood, with most of the injected peptide reaching the brain intact. Furthermore, emerging evidence indicates that GLP-1R stimulation facilitates hippocampal synaptic plasticity, cognition, and cell survival (123-125). Exendin-4 was recently found to block $A \beta$ oligomer-induced impairment in insulin signaling in hippocampal cultured neurons (10). Exendin-4 and liraglutide also restored impaired insulin signaling in the brains of a trans- 
A Healthy aging

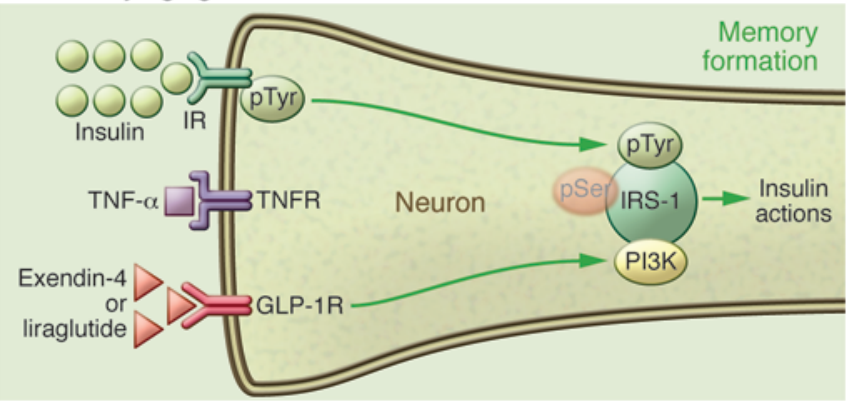

B Early $A D$

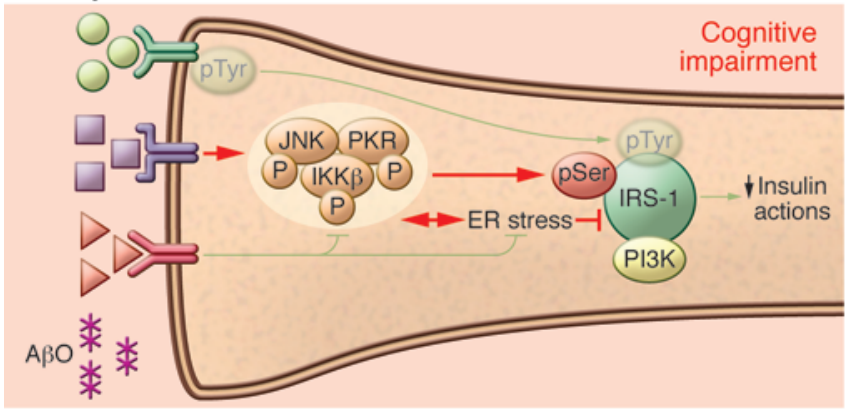

D Late $A D$

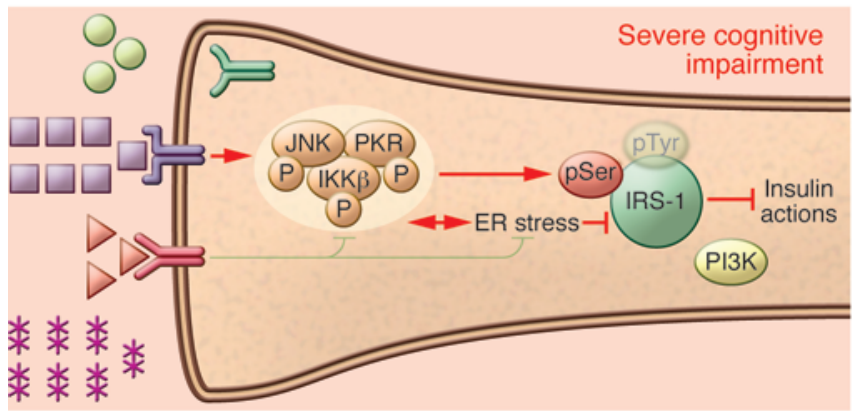

C Boosting insulin signaling in early $A D$

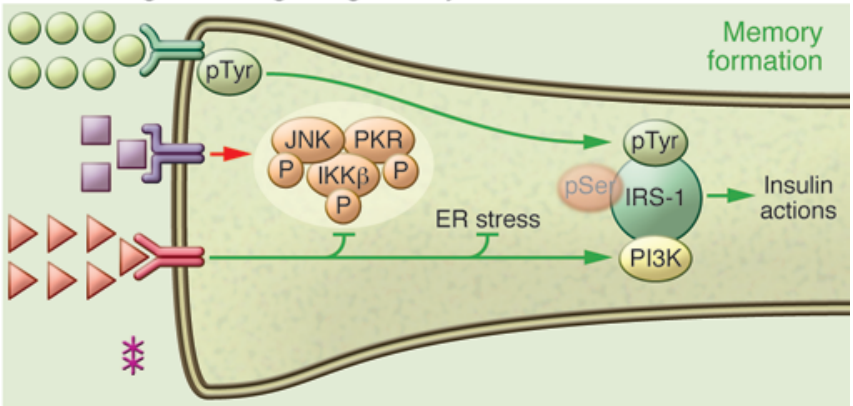

E Boosting insulin signaling in late $A D$

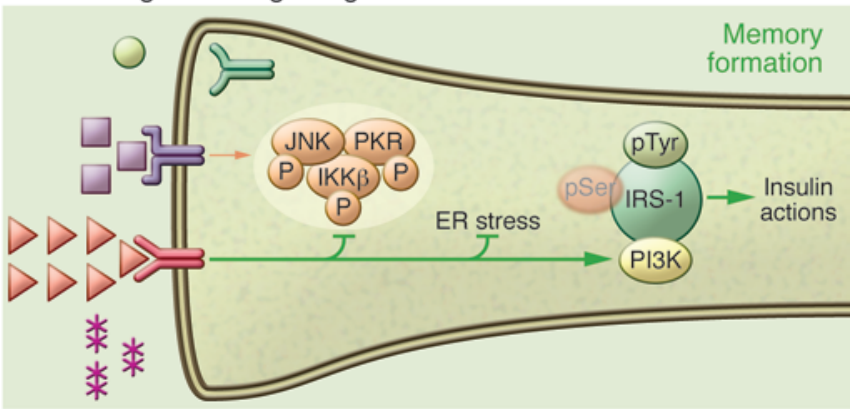

Figure 3

Boosting brain insulin signaling to combat AD. (A) In healthy aging, IRs are present at synapses, and proper insulin signaling favors synapse function and leads to memory formation. (B) In early AD, accumulation of A $\beta O$ s stimulates TNF- $\alpha$ signaling, which activates the JNK pathway (11, 54) and, possibly, PKR and IKK pathways. Activation of these stress-sensitive kinases, which can also be triggered by ER stress in peripheral tissues (69), results in IRS-1pSer, decreasing downstream insulin signaling (11, 31, 54). This contributes to initial cognitive impairment in early AD. (C) At later $\mathrm{AD}$ stages, increased accumulation of $\mathrm{A} \beta \mathrm{O}$ s and their binding to synapses lead to removal of IRs from the neuronal plasma membrane (14, $15,47)$. Additionally, TNF- $\alpha / J N K$ activation ultimately blocks insulin actions (11), contributing to severe impairment in cognition and memory. (D) Stimulation of insulin and GLP-1Rs blocks early A $\beta O$-induced defects in insulin signaling. Insulin protects neurons by preventing $A \beta O$ binding to neurons (15). In addition, activation of GLP-1Rs by exendin-4 or liraglutide and of IRs by insulin prevents JNK activation, allowing physiological tyrosine phosphorylation of IRS-1 and stimulating downstream insulin signaling. (E) At later AD stages, activation of GLP-1Rs prevents inhibitory IRS-1pSer, stimulating insulin-related downstream signaling pathways and ameliorating cognitive and memory impairment. Red arrows indicate inhibitory pathways and green arrows indicate stimulatory pathways of insulin signaling.

genic mouse model of $\mathrm{AD}$, improving cognition and decreasing $A \beta$ accumulation $(10,126)$. As recently suggested, an agent that chronically decreases $A \beta$ levels should be beneficial in APOE $\varepsilon 4$ carriers (127). If the beneficial effect of GLP-1R agonists is found to translate to primates, APOE $\varepsilon 4$ carriers may possibly benefit from the use of GLP-1R agonists. GLP-1R activation may thus provide a novel strategy to resensitize impaired brain insulin signaling and prevent or halt neurodegeneration in $\mathrm{AD}$.

In conclusion, the link between $\mathrm{AD}$ and diabetes may have profound implications for our understanding of the mechanisms underlying neuronal dysfunction in AD. Indeed, novel effective and safe therapeutic opportunities for AD are likely to arise from efforts aimed at unraveling the mechanisms that account for brain insulin resistance, as well as from a deeper understanding of the connection between AD and diabetes (Figure 3) $(21,27)$. The upcoming results from recently implemented clinical trials using both insulin (128) and GLP-1R agonists in AD are highly anticipated in the field. These studies will hopefully demonstrate a benefit for $\mathrm{AD}$ therapeutics targeting insulin signaling to treat this devastating disease. 


\section{Acknowledgments}

Work in the author's laboratory was supported by grants from Human Frontiers Science Program (HFSP) and the John Simon Guggenheim Memorial Foundation; the Brazilian funding agencies Conselho Nacional de Desenvolvimento Científico e Tecnológico (CNPq); Fundação de Amparo à Pesquisa do Estado do Rio de Janeiro (FAPERJ); and National Institute for Translational Neuroscience. I thank Sergio T. Ferreira (Federal University of Rio de Janeiro) for critical reading and thoughtful discus- sions, and Denise G. De Felice (M.Sc.) for assistance with text editing. I also thank Mychael V. Lourenco (M.Sc.), Marcelo N. Vieira, and Jordano B. Moreira (M.Sc.) for their help with preparation of the figures.

Address correspondence to: Fernanda G. De Felice, Institute of Medical Biochemistry, CCS, Room H2-019, Federal University of Rio de Janeiro, Rio de Janeiro, RJ 21944-590, Brazil. Phone: 5521.2562.6515; Fax: 5521.2270.8647; E-mail: felice@bioqmed.ufrj.br.
1. Banting FG, Best CH, Coll JB, Campbell WR, Flechter AA. Pancreatic extracts in the treatment of diabetes mellitus. Can Med Assoc J. 1922;12(3):141-146.

2. Woods SC, Lotter EC, McKay LD, Porte D. Chronic intracerebroventricular infusion of insulin reduces food intake and body weight of baboons. Nature. 1979;282(5738):503-505.

3. Marino JS, Xu Y, Hill JW. Central insulin and leptin-mediated autonomic control of glucose homeostasis. Trends Endocrinol Metab. 2011;22(7):275-285.

4. Scherer $\mathrm{T}$, et al. Brain insulin controls adipose tissue lipolysis and lipogenesis. Cell Metab. 2011; 13(2):183-194.

5. Zhao WQ, Chen H, Quon MJ, Alkon DL. Insulin and the insulin receptor in experimental models of learning and memory. Eur J Pharmacol. 2004; 490(1-3):71-81.

6. Zhao WQ, Alkon DL. Role of insulin and insulin receptor in learning and memory. Mol Cell Endocrinol. 2001;177(1-2):125-134.

7. Plum L, Schubert M, Brüning JC. The role of insulin receptor signaling in the brain. Trends Endocrinol Metab. 2005;16(2):59-65.

8. Kovacs P, Hajnal A. In vivo electrophysiological effects of insulin in the rat brain. Neuropeptides. 2009;43(4):283-293.

9. Ott V, Benedict C, Schultes B, Born J, Hallschmid $M$. Intranasal administration of insulin to the brain impacts cognitive function and peripheral metabolism. Diabetes Obes Metab. 2012;14(3):214-221.

10. Bomfim TR, et al. An anti-diabetes agent protects the mouse brain from defective insulin signaling caused by Alzheimer's disease-associated A $\beta$ oligomers. J Clin Invest. 2012;122:1339-1353.

11. Wan $Q$, et al. Recruitment of functional GABA-A receptors to postsynaptic domains by insulin. Nature. 1997;388(6643):686-690.

12. Biessels GJ, et al. Place learning and hippocampal synaptic plasticity in streptozotocin-induced diabetic rats. Diabetes. 1996;45(9):1259-1266.

13. Li L, Hölscher C. Common pathological processes in Alzheimer disease and type 2 diabetes: a review. Brain Res Rev. 2007;56(2):384-402.

14. Fernandez AM, Torres-Alemán I. The many faces of insulin-like peptide signalling in the brain. Nat Rev Neurosci. 2012;13(4):225-239.

15. Zhao WQ, et al. Amyloid beta oligomers induce impairment of neuronal insulin receptors. FASEBJ. 2008;22(1):246-260

16. De Felice FG, et al. Protection of synapses against Alzheimer's-linked toxins: insulin signaling prevents the pathogenic binding of A $\beta$ oligomers. Proc Natl Acad Sci U S A. 2009;106(6):1971-1976.

17. Chiu S-L, Chen C-M, Cline HT. Insulin receptor signaling regulates synapse number, dendritic plasticity, and circuit function in vivo. Neuron. 2008; 58(5):708-719.

18. Nelson TJ, Sun M-K, Hongpaisan J, Alkon DL. Insulin, PKC signaling pathways and synaptic remodeling during memory storage and neuronal repair. Eur J Pharmacol. 2008;585(1):76-87.

19. Benedict $C$, et al. Intranasal insulin improves memory in humans. Psychoneuroendocrinology. 2004; 29(10):1326-1334.

20. Reger MA, et al. Intranasal insulin improves cogni- tion and modulates $\beta$-amyloid in early AD. Neurology. 2008;70(6):440-448.

21. Craft S, et al. Intranasal insulin therapy for Alzheimer disease and amnestic mild cognitive impairment: a pilot clinical trial. Arch Neurol. 2012; 69(1):29-38.

22. Schubert $M$, et al. Role for neuronal insulin resistance in neurodegenerative diseases. Proc Natl Acad Sci U S A. 2004;101(9):3100-3105.

23. Craft $S$, Watson G. Insulin and neurodegenerative disease: shared and specific mechanisms. Lancet Neurol. 2004;3(3):169-178.

24. Ott A, Stolk RP, Hofman A, van Harskamp F, Grobbee DE, Breteler MMB. Association of diabetes mellitus and dementia: The Rotterdam Study. Diabetologia. 1996;39(11):1392-1397.

25. Steen E, et al. Impaired insulin and insulin-like growth factor expression and signaling mechanisms in Alzheimer's disease - is this type 3 diabetes? J Alzheimers Dis. 2005;7(1):63-80.

26 . Hoyer S, Nitsch R. Cerebral excess release of neurotransmitter amino acids subsequent to reduced cerebral glucose metabolism in early-onset dementia of Alzheimer type. J Neural Transm. 1989;75(3):227-232.

27. Craft S. Alzheimer disease: insulin resistance and $\mathrm{AD}-$ extending the translational path. Nat Rev Neurol. 2012;8(7):360-362.

28. Takeda S, et al. Diabetes-accelerated memory dysfunction via cerebrovascular inflammation and $A \beta$ deposition in an Alzheimer mouse model with diabetes. Proc Natl Acad Sci U S A. 2010;107(15):7036-7041.

29. Salkovic-Petrisic M, Hoyer S. Central insulin resistance as a trigger for sporadic Alzheimer-like pathology: an experimental approach. J Neural Transm. 2007;72(72):217-233.

30. Talbot K, et al. Demonstrated brain insulin resistance in Alzheimer's disease patients is associated with IGF-1 resistance, IRS-1 dysregulation, and cognitive decline. J Clin Invest. 2012;122(4):1316-1338.

31. Ballard C, Gauthier S, Corbett A, Brayne C, Aarsland D, Jones E. Alzheimer's disease. Lancet. 2011; 377(9770):1019-1031.

32. Danaei G, et al. National, regional, and global trends in fasting plasma glucose and diabetes prevalence since 1980: systematic analysis of health examination surveys and epidemiologival studies with 370 country-years and 2.7 million participants. Lancet. 2011;378(9785):31-40.

33. Alzheimer A, Stelzmann RA, Schnitzlein HN, Murtagh FR. An English translation of Alzheimer's 1907 paper "Uber eine eigenartige Erlranliung der Hirnrinde”. Clin Anat. 1995;8(6):429-431.

34. Glenner GG, Wong CW. Alzheimer's disease: initial report of the purification and characterization of a novel cerebrovascular amyloid protein. Biochem Biophys Res Commun. 1984;120(3):885-890.

35. Masters CL, Simms G, Weinman NA, Multhaup G, McDonald BL, Beyreuther K. Amyloid plaque core protein in Alzheimer disease and Down syndrome. Proc Natl Acad Sci U S A. 1985;82(12):4245-4249.

36. Negash S, Bennett DA, Wilson RS, Schneider JA, Arnold SE. Cognition and neuropathology in aging: multidimensional perspectives from the Rush Religious Orders Study and Rush Memory and Aging Project. Curr Alzheimer Res. 2011;8(4):336-340.
37. Terry RD, et al. Physical basis of cognitive alterations in Alzheimer disease: synapse loss is the major correlate of cognitive impairment. Ann Neurol. 1991;30(4):572-580.

38. Lambert MP, et al. Diffusible, nonfibrillar ligands derived from Abeta1-42 are potent central nervous system neurotoxins. Proc Natl Acad Sci U S A. 1998; 95(11):6448-6453.

39. Björklund NL, Reese LC, Sadagoparamanujam VM, Ghirardi V, Woltjer RL, Taglialatela G. Absence of amyloid $\beta$ oligomers at the postsynapse and regulated synaptic $\mathrm{Zn}^{2+}$ in cognitively intact aged individuals with Alzheimer's disease neuropathology. Mol Neurodegener. 2012;7(1):23.

40. Gong Y, et al. Alzheimer's disease-affected brain: presence of oligomeric Abeta ligands (ADDLs) suggests a molecular basis for reversible memory loss. Proc Natl Acad Sci U S A. 2003;100(18):10417-10422.

41. Xia W, et al. A specific enzyme-linked immunosorbent assay for measuring $\beta$-amyloid protein oligomers in human plasma and brain tissue of patients with Alzheimer disease. Arch Neurol. 2009; 66(2):190-199.

42. Fukumoto $H$, et al. High-molecular-weight $\beta$-amyloid oligomers are elevated in cerebrospinal fluid of Alzheimer patients. FASEBJ. 2010;24(8):2716-2726.

43. Ferreira ST, Vieira MNN, De Felice FG. Soluble protein oligomers as emerging toxins in Alzheimer's and other amyloid diseases. IUBMB Life. 2007;59(4-5):332-345.

44. Ferreira ST, Klein WL. The A $\beta$ oligomer hypothesis for synapse failure and memory loss in Alzheimer's disease. Neurobiol Learn Mem. 2011;96(4):529-543.

45. Haass C, Selkoe DJ. Soluble protein oligomers in neurodegeneration: lessons from the Alzheimer's amyloid $\beta$-peptide. Nat Rev Mol Cell Biol. 2007; 8(2):101-112.

46. Moloney AM, Griffin RJ, Timmons S, O’Connor R, Ravid R, O'Neill C. Defects in IGF-1 receptor, insulin receptor and IRS- $1 / 2$ in Alzheimer's disease indicate possible resistance to IGF-1 and insulin signalling. Neurobiol Aging. 2010;31(2):224-243.

47. Lacor PN, et al. A $\beta$ oligomer-induced aberrations in synapse composition, shape, and density provide a molecular basis for loss of connectivity in Alzheimer's disease. J Neurosci. 2007;27(4):796-807.

48. Snyder EM, et al. Regulation of NMDA receptor trafficking by amyloid- $\beta$. Nat Neurosci. 2005; 8(8):1051-1058.

49. Jurgensen S, et al. Activation of D1/D5 dopamine receptors protects neurons from synapse dysfunction induced by amyloid- $\beta$ oligomers. J Biol Chem. 2011;286(5):3270-3276

50. De Felice FG, et al. Alzheimer's disease-type neuronal tau hyperphosphorylation induced by Abeta oligomers. Neurobiol Aging. 2008;29(9):1334-1347.

51. Zempel H, Thies E, Mandelkow E, Mandelkow EM. A $\beta$ oligomers cause localized $\mathrm{Ca}^{2+}$ elevation, missorting of endogenous tau into dendrites, tau phosphorylation and destruction of microtubules and spines. J Neurosci. 2010;30(36):11938-11950.

52. Ma QL, et al. $\beta$-amyloid oligomers induce phosphorylation of tau and inactivation of insulin receptor substrate via c-Jun $\mathrm{N}$-terminal kinase signaling: suppression by omega-3 fatty acids and curcumin. 
J Neurosci. 2009;29(28):9078-9089.

53. De Felice FG, et al. A $\beta$ oligomers induce neuronal oxidative stress through an N-methyl-D-aspartate receptor-dependent mechanism that is blocked by the Alzheimer drug memantine. J Biol Chem. 2007; 282(15):11590-11601.

54 . Saraiva LM, et al. Amyloid- $\beta$ triggers the release of neuronal hexokinase 1 from mitochondria. PLoS One. 2010;5(12):e15230.

55. Alberdi E, et al. Amyloid $\beta$ oligomers induce $\mathrm{Ca}^{2+}$ dysregulation and neuronal death through activation of ionotropic glutamate receptors. Cell Calcium. 2010;47(3):264-272.

56. White MF. IRS proteins and the common pathway to diabetes. Am J Physiol Endocrinol Metab. 2002; 283(3):E413-E422.

57. Boura-Halfon S, Zick Y. Phosphorylation of IRS proteins, insulin action and insulin resistance. $A m$ J Physiol Endocrinol Metab. 2009;296(4):E581-E591.

58. Haj-ali V, Mohaddes G, Babri SH. Intracerebroventricular insulin improves spatial learning and memory in male Wistar rats. Behav Neurosci. 2009; 123(6):1309-1314.

59. Irvine EE, et al. Insulin receptor substrate 2 is a negative regulator of memory formation. Learn Mem 2011;18(6):375-383.

60. Killick R, et al. Deletion of Irs2 reduces amyloid deposition and rescues behavioural deficits in APP transgenic mice. Biochem Biophys Res Commun. 2009; 386(1):257-262.

61. Taguchi A, White MF. Insulin-like signaling, nutrient homeostasis, and life span. Annu Rev Physiol. 2008;70:191-212.

62. Freude S, et al. Neuronal IGF-1 resistance reduces $A \beta$ accumulation and protects against premature death in a model of Alzheimer's disease. FASEB J. 2009;23(10):3315-3324.

63 . Hirosumi J, et al. A central role for JNK in obesity and insulin resistance. Nature. 2002;420(6913):333-336.

64. Pessin JE, Saltiel AR. Signaling pathways in insulin action: molecular targets of insulin resistance. J Clin Invest. 2000;106(2):165-169.

65. Lee YH, White MF. Insulin receptor substrate proteins and diabetes. Arch Pharm Res. 2004;27(4):361-370.

66. Puig KL, Floden AM, Adhikari R, Golovko MY, Combs CK. Amyloid precursor protein and proinflammatory changes are regulated in brain and adipose tissue in a murine model of high-fat diet-induced obesity. PloS One. 2012;7(1):e30378.

67. Nakamura T, et al. Double-stranded RNA-dependent protein kinase links pathogen sensing with stress and metabolic homeostasis. Cell. 2010; 140(3):338-348.

68. Yang J, Park Y, Zhang H, Xu X, Laine GA, Dellsperger $\mathrm{KC}$, Zhang $\mathrm{C}$. Feed-forward signaling of TNFalpha and NF-kappaB via IKK-beta pathway contributes to insulin resistance and coronary arteriolar dysfunction in type 2 diabetic mice. Am J Physio Heart Circ Physiol. 2009;296(6):H1850-H1858.

69. Hoozemans JJM, van Haastert ES, Nijholt DAT, Rozemuller AJM, Eikelenboom P, Scheper W. The unfolded protein response is activated in pretangle neurons in Alzheimer's disease hippocampus. AmJ Pathol. 2009;174(4):1241-1251.

70. Serrano F, Klann E. Reactive oxygen species and synaptic plasticity in the aging hippocampus. Ageing Res Rev. 2004;3(4):431-443.

71. Cheng Z, Tseng Y, White MF. Insulin signaling meets mitochondria in metabolism. Trends Endocrinol Metab. 2010;21(10):589-598.

72. Reddy VP, Zhu X, Perry G, Smith MA. Oxidative stress in diabetes and Alzheimer disease. J Alzheimers Dis. 2009;16:763-774.

73. Manolopoulos KN, Klotz LO, Korsten P, Bornstein SR, Barthel A. Linking Alzheimer's disease to insulin resistance: the FoxO response to oxidative stress. Mol Psychiatry. 2010;15(11):1046-1052.

74. Paula-Lima AC, et al. Amyloid $\beta$-peptide oligomers stimulate RyR-mediated $\mathrm{Ca}^{2+}$ release inducing mitochondrial fragmentation in hippocampal neurons and prevent RyR-mediated dendritic spine remodeling produced by BDNF. Antioxid Redox Signal. 2011;14:1209-1223.

75. Picone $P$, et al. Insulin-activated Akt rescues $A \beta$ oxidative stress-induced cell death by orchestrating molecular trafficking. Aging Cell. 2011;10(5):832-843.

76. Decker H, et al. N-methyl-D-aspartate receptors are required for synaptic targeting of Alzheimer's toxic A $\beta$ oligomers. J Neurochem. 2010;115(6):1520-1529.

77. Hardy JA, Selkoe DJ. The amyloid hypothesis of Alzheimer disease: progress and problems on the road to therapeutics. Science. 2002;297(5580):353-356.

78. Bertram L, Lill CM, Tanzi RE. The genetics of Alzheimer's disease: back to the future. Neuron. 2010; 68(2):270-281.

79. Hotamisligil GS. Endoplasmic reticulum stress and the inflammatory basis of metabolic disease. Cell. 2010;140(6):900-917.

80. Craft S. The role of metabolic disorders in Alzheimer disease and vascular dementia: two roads converged. Arch Neurol. 2009;66(3):300-305.

81. De La Monte S. Metabolic derangements mediate cognitive impairment and Alzheimer's disease: role of peripheral insulin-resistance diseases. Panminerva Med. 2012;54(3):171-178.

82. Zimmet $\mathrm{P}$, et al. The metabolic syndrome in children and adolescents. Lancet. 2007;369(9579):2059-2061.

83. Mattson MP. Energy intake and exercise as determinants of brain health and vulnerability to injury and disease. Cell Metab. 2012;16(6):706-722.

84. Stranahan AM, et al. Diet-induced insulin resistance impairs hippocampal synaptic plasticity and cognition in middle-aged rats. Hippocampus. 2008; 18(11):1085-1088.

85. Tasali E, Leproult R, Ehrmann DA, Van Cauter E. Slow-wave sleep and the risk of type 2 diabetes in humans. Proc Natl Acad Sci U S A. 2008; 105(3):1044-1049.

86. Kang J, et al. Amyloid- $\beta$ dynamics are regulated by orexin and the sleep-wake cycle. Science. 2009; 326(5955):1005-1007.

87. Barlow KM, Thomson E, Johnson D, Minns RA Late neurologic and cognitive sequelae of inflicted traumatic brain injury in infancy. Pediatrics. 2005;116(1):174-185

88. Uryu K, et al. Repetitive mild brain trauma accelerates $A \beta$ deposition, lipid peroxidation and cognitive impairment in a transgenic mouse model of Alzheimer amyloidosis. J Neurosci. 2002;22(2):446-454.

89. Yoshiyama Y, et al. Enhanced neurofibrillary tangle formation, cerebral atrophy and cognitive deficits induced by repetitive mild brain injury in a transgenic tauopathy mouse model. J Neurotrauma. 2005;22(10):1134-1141

90. Huh JW, Widing AG, Raghupathi R. Differential effects of injury severity on cognition and cellular pathology after contusive brain trauma in the immature rat. J Neurotrauma. 2011;28(2):245-257.

91. Johnson VE, Stewart W, Smith DH. Traumatic brain injury and amyloid- $\beta$ pathology: a link to Alzheimer's disease? Nat Rev Neurosci. 2010;11(5):361-370.

92. Cole GM, Frautschy SA. The role of insulin and neurotrophic factor signaling in brain aging and Alzheimer's disease. Exp Gerontol. 2007;42(1-2):10-21.

93. Craft S, Peskind E, Schwartz MW, Schellenberg GD, Raskind M, Porte D. Cerebrospinal fluid and plasma insulin levels in Alzheimer's disease: relationship to severity of dementia and apolipoprotein E genotype. Neurology. 1998;50(1):164-168.

94. Fujisawa Y, Sasaki K, Akiyama K. Increased insulin levels after OGTT load in peripheral blood and cerebrospinal fluid of patients with dementia of Alzheimer type. Biol Psychiatry. 1991;30(12):1219-1228.

95. Gil-Bea FJ, et al. Insulin levels are decreased in the cerebrospinal fluid of women with prodromal Alzheimer's disease. J Alzheimers Dis. 2010;22(2):405-413.
96. Bayer-Carter JL, et al. Diet intervention and cerebrospinal fluid biomarkers in amnestic mild cognitive impairment. Arch Neurol. 2011;68(6):743-752.

97. Benedict $C$, et al. Impaired insulin sensitivity as indexed by the HOMA score is associated with deficits in verbal fluency and temporal lobe gray matter volume in the elderly. Diabetes Care. 2012;35(3):488-494.

98. Gregor MF, Hotamisligil GS. Inflammatory mechanisms in obesity. Annu Rev Immunol. 2011;29:415-445.

99. Clark I, Atwood C, Bowen R, Paz-Filho G, Vissel B. Tumor necrosis factor-induced cerebral insulin resistance in Alzheimer's disease links numerous treatment rationales. Pharmacol Rev. 2012; 64(4):1004-1026

100.Samuel VT, Shulman GI. Mechanisms for insulin resistance: common threads and missing links. Cell. 2012;148(5):852-871.

101. Fu S, et al. Aberrant lipid metabolism disrupts calcium homeostasis causing liver endoplasmic reticulum stress in obesity. Nature. 2011;473(7348):528-531.

102. Holzer RG, et al. Saturated fatty acids induce c-Src clustering within membrane subdomains, leading to JNK activation. Cell. 2011;147(1):173-184.

103. Karmi A, et al. Increased brain fatty acid uptake in metabolic syndrome. Diabetes. 2010;59(9):2171-2177.

104.Geekiyanage H, Chan C. MicroRNA-137/181c regulates serine palmitoyltransferase and in turn amyloid- $\beta$, novel targets in sporadic Alzheimer's disease. J Neurosci. 2011;31:14820-14830.

105. Fassbender K, et al. Simvastatin strongly reduces levels of Alzheimer's disease $\beta$-amyloid peptides Abeta 42 and Abeta 40 in vitro and in vivo. Proc Natl Acad Sci U S A. 2001;98(10):5856-5861.

106. Di Paolo G, Kim T-W. Linking lipids to Alzheimer's disease: cholesterol and beyond. Nat Rev Neurosci. 2011;12(5):284-296

107. Perry VH, Nicoll JAR, Holmes C. Microglia in neurodegenerative disease. Nat Rev Neurol. 2010; 6(4):193-201.

108. Herrup K. Reimagining Alzheimer's disease - an age-based hypothesis. J Neurosci. 2010; 30(50):16755-16762.

109. O'Connor T, et al. Phosphorylation of the translation initiation factor eIF2 $\alpha$ increases BACE1 levels and promotes amyloidogenesis. Neuron. 2008; 60(6):988-1009

110. Cai D, Liu T. Hypothalamic inflammation: a double-edged sword to nutritional diseases. Ann NY Acad Sci. 2011;1243:E1-E39.

111.Zhang X, Zhang G, Zhang H, Karin M, Bai H, Cai D. Hypothalamic IKK $\beta / \mathrm{NF}-\kappa \mathrm{B}$ and ER stress link overnutrition to energy imbalance and obesity. Cell. 2008;135(1):61-73.

112. Hanson LR, Frey WH. Intranasal delivery bypasses the blood-brain barrier to target therapeutic agents to the central nervous system and treat neurodegenerative disease. BMC Neurosci. 2008;9(suppl 3):S5.

113. Benedict $C$, et al. Intranasal insulin enhances postprandial thermogenesis and lowers postprandial serum insulin levels in healthy men. Diabetes. 2010;60(1):114-118.

114. Benedict C, Kern W, Schultes B, Born J, Hallschmid M. Differential sensitivity of men and women to anorexigenic and memory-improving effects of intranasal insulin. J Clin Endocrinol Metab. 2008; 93(4):1339-1344.

115.Lasagna-Reeves CA, Clos AL, Midoro-Hiriuti T, Goldblum RM, Jackson GR, Kayed R. Inhaled insulin forms toxic pulmonary amyloid aggregates. Endocrinology. 2010;151(10):4717-4724.

116. Shemesh E, Rudich A, Harman-Boehm I, Cukierman-Yaffe T. Effect of intranasal insulin on cognitive function: a systematic review. J Clin Endocrinol Metab. 2011;97(2):366-376.

117. Reger MA, et al. Effects of intranasal insulin on cognition in memory-impaired older adults: modulation by APOE genotype. Neurobiol Aging. 2006; 27(3):451-458. 
118. Townsend M, Mehta T, Selkoe DJ. Soluble Abeta inhibits specific signal transduction cascades common to the insulin receptor pathway. J Biol Chem. 2007;282(46):33305-33312.

119. Schiöth HB, Craft S, Brooks SJ, Frey WH 2nd, Benedict C. Brain insulin signaling and Alzheimer's disease: current evidence and future directions. $\mathrm{Mol}$ Neurobiol. 2012;46(1):4-10.

120.Vilsbøll T, Krarup T, Madsbad S, Holst JJ. Both GLP-1 and GIP are insulinotropic at basal and postprandial glucose levels and contribute nearly equally to the incretin effect of a meal in healthy subjects. Regul Pept. 2003;114(2-3):115-121.
121. Mattson MP, Perry T, Greig NH. Learning from the gut. Nat Med. 2003;9(9):1113-1115.

122. Göke R, Larsen PJ, Mikkelsen JD, Sheikh SP. Distribution of GLP-1 binding sites in the rat brain: evidence that exendin- 4 is a ligand of brain GLP- 1 binding sites. European J Neurosci. 2005;7(11):2294-2300.

123. During MJ, et al. Glucagon-like peptide-1 receptor is involved in learning and neuroprotection. Nat Med. 2003;9(9):1173-1179.

124.Abbas T, Faivre E, Hölscher C. Impairment of synaptic plasticity and memory formation in GLP-1 receptor $\mathrm{KO}$ mice: interaction between type 2 diabetes and Alzheimer's disease. Behav Brain Res. 2009;
205(1):265-271.

125.Yusta B, et al. GLP-1 receptor activation improves $\beta$-cell function and survival following induction of endoplasmic reticulum stress. Cell Metab. 2006; 4(5):391-406

126. McClean PL, Parthsarathy V, Faivre E, Holscher C. The diabetes drug liraglutide prevents degenerative processes in a mouse model of Alzheimer's disease. J Neurosci. 2011;31(17):6587-6594.

127. Selkoe DJ. Preventing Alzheimer's disease. Science. 2012;337(6101):1488-1492.

128. Wadman M. US government sets out Alzheimer's plan. Nature. 2012;485(7399):426-427. 